\title{
Link between Allais Effect and General Relativity's Residual Arc during Solar Eclipse
}

\author{
Russell Bagdoo \\ Saint-Bruno-de-Montarville, Quebec, Canada \\ Email:rbagdoo@yahoo.ca,rbagdoo@gmail.com
}

How to cite this paper: Bagdoo, R. (2020) Link between Allais Effect and General Relativity's Residual Arc during Solar Eclipse. Journal of Modern Physics, 11, 1620-1638. https://doi.org/10.4236/jmp.2020.1110101

Received: September 4, 2020

Accepted: October 23, 2020

Published: October 26, 2020

Copyright $\odot 2020$ by author(s) and Scientific Research Publishing Inc. This work is licensed under the Creative Commons Attribution International License (CC BY 4.0).

http://creativecommons.org/licenses/by/4.0/

\section{(c) (i) Open Access}

\begin{abstract}
The purpose of this article is to establish a relation between two gravitational anomalies: one that has attracted part of the scientific community, the Allais effect that occurs during solar eclipse; the other, noticed but forgotten by the whole scientific community, the General Relativity's residual arc of the curvature of rays of light in the solar gravitational field during the same eclipse. There is a systematically observed deflection about $10 \%$ larger than the theoretical value of General Relativity, which coincides with the "eclipse effect" found by Maurice Allais, thrown aside because it upsets the established truths. These corresponding anomalies were never explained by any theories and turn out to be new gravitational physics.
\end{abstract}

\section{Keywords}

Allais Effect, Paraconical Pendulum, General Relativity, Eclipse Experience, Parallax, Arc Residue, Interferometry

\section{Introduction}

\subsection{Preliminaries}

Based on the equivalence principle, Einstein revealed a deep relation of gravity to the geometry of spacetime. General Relativity (GR) has undergone an impressive series of confirmations mostly regarding "strong" fields. But in "weak" fields where GR does not distinguish from the Newtonian limit, there are unexplained phenomena like galaxy rotation curves, the Pioneer anomaly. There is little direct evidence that conventional theories of gravity are correct on large scales. Despite all the success of Newtonian gravity and GR on the scale of the solar system, data of unique precision collected for the last two decades by satellite-based telescopes covering all frequencies and digital image processing gave a 
number of results where these theories run into problems, such as the unexpected secular increase of the Astronomical Unit or the abnormal change in acceleration for flybys of spacecraft. We emphasize the scientific aspect of peculiar movements exhibited by an anisotropic paraconical pendulum at the time of a solar eclipse in 1954.

The aim of this article is to establish a relation between the abnormal deviation of the pendulum (Allais effect) during this solar eclipse and the observed residual arc of the curvature of the rays of light in the solar gravitational field (GR) during the same eclipse. Both gravitational anomalies were never explained by GR or by any other theories and it raises question about their nature. Our approach is as follows. In Section 1, we describe the gravitational deflection of light by the Sun during solar eclipses insisting on the fact that many observations gave a deflection $10 \%$ larger than the theoretical value during eclipse experience. We present from the outset the Allais effect, first reported in 1954 by Maurice Allais, which is an anomalous precession of the plane of oscillation of a paraconical pendulum during a solar eclipse. In Section 2, we make a mathematical link between the unexpected turn that the pendulum took during the eclipse, changing its angle of rotation by $13.5^{\circ}$, and the about $10 \%$ arc's deviation observed during the same eclipse experience. Although it is not the task of this paper, we also speculate on modification of Einsteinian gravitational mechanics and a discussion mentions briefly that these anomalies turn out to be new gravitational physics including "antigravity". In Section 3, the fact that an exact agreement between theory and measurements has been obtained by radio interferometry, but has never been obtained by eclipse technique, indicating a genuine insufficiency of Einstein's theory during eclipse. In Section 4, we discuss two manners to experiment the behaviour of the gravity: measure the angle of deflection of light and measure the delay time of signals. In Section 5, we show three possible cosmological consequences of the Allais effect linked to GR's residual arc during total solar eclipse. Conclusion in Section 6: during the eclipse, the unexplained excess of arc of general relativity would be consistent with the abrupt deviation of the oscillation plane of the Allais pendulum; for these two phenomena, the currently accepted physical theories offer no explanation for this slight deviation from gravitational laws, which suggest a new physics.

\subsection{Many Observations Gave a Deflection $10 \%$ Larger Than the Theoretical Value of General Relativity during Eclipse Experience}

The first test of Einstein's prediction was the apparent bending of light as it passes near a massive body [1]. This effect was conclusively observed during the solar eclipse of May 29, 1919, when the Sun was silhouetted against the Hyades star cluster, for which the positions were well known. It was mainly made through the initiative of the British astronomer Eddington. He was stationed on an island off the western coast of Africa and sent another group of British scientists to Brazil. Their measurements of several of the stars in the cluster showed 
that the light from these stars was indeed bent as it grazed the Sun. The result obtained by measuring the plates $(1.98 " \pm 0.12 "$ and $1.61 " \pm 0.31 ")$ confirmed almost the exact displacement of Einstein's predictions [2].

GR predicts that locally straight lines that pass near the Sun are bent relatively to the straight lines that pass far from the Sun in completely empty space by an additional 0.875 arcsecond. Thus, the total deflection must be $\sim 1.75$ arcseconds. The apparent displacement of light results from the warping of space in the vicinity of the massive object through which light travels. The light never changes course, but merely follows the curvature of space. Astronomers now refer to this displacement of light as gravitational lensing. But the Sun's gravity is relatively weak compared with what's out there in the depths of space. As a dramatic example of gravitational lensing, the light from a quasar (a young, distant galaxy that emits prodigious amounts of radio energy) 8 billion light years away is bent round by the gravity of a closer galaxy that's "only" 400 million light years distant from Earth.

Although the eclipse results distinguished clearly among the possibilities of no deflection, the Newtonian deflection, and the Einsteinian deflection, their relatively large experimental errors made it important to repeat the measurements. Since that time, measurements of the deflection of the light by the Sun, although they are difficult, have been made at a number of around 400 total eclipses with only modest improvement over previous eclipse measurements. The values were anywhere between three-quarters and one and one-half times the general relativistic prediction: many observations gave a deflection $10 \%$ larger than the theoretical value. The deviation between theory and measurements is too high to be only due to errors of observation [2] [3].

Even if there is no doubt that GR is nearer the truth than the value obtained from Newtonian mechanics or any other theory so far proposed, even if radio interferometry now replaced the eclipse technique bringing an almost exact agreement between theory and measurements (which has never been obtained by eclipse technique), this deviation during eclipse indicates a genuine insufficiency of Einstein's theory.

\subsection{Allais Effect; An Experience of Great Precision}

Professor Maurice Allais is a French physicist, winner of the 1988 Nobel Prize in Economics, winner of the 1959 Galabert Prize of the French Astronautical Society, and also a laureate of the United States Gravity Research Foundation due to his gravitational experiments. In the 1950s he undertook several experimental series in Paris which involved repeated determinations of the rate of precession of a paraconical pendulum which he had invented. Suspended via a small steel ball bearing, this pendulum with anisotropic support has this peculiarity to be able to raise abruptly the oscillation plane when there is a sudden disturbance. 7 series of experiments succeeded one another: 32 days in June and July 1954; 9 in September 1954; 37 in November and December 1954; and in 1955, 7, 15, 30 and 
17 days. He released his pendulum every 20 minutes-for more security, the pendulum was stopped vertically, then re-tautened-and recorded the azimuth every minute for 14 minutes. There were thus 72 series of experiments by 24 hours without missing a data point. He detected various periodic anomalies in the motion of this pendulum by using elaborate statistical analysis. One of these experimental series happened to overlap with the eclipse of Sun of June 30th, 1954 [4] [5] [6].

During the eclipse, M. Allais put in evidence an unexpected disturbance of the effect of Foucault. At the exact onset of the eclipse, the plane of the oscillation got up abruptly of $4.5^{\circ}$.

Twenty minutes before the maximum of the eclipse, it reached $13.5^{\circ}$ to decline slowly until an abnormality of $1^{\circ}$ at the end of the phenomenon. This unexpected large scale excursion in the angular plane persisted throughout the length of the eclipse, a total of 2.5 hours of observations from eclipse start on Earth's west limb to end on the east limb. Both before and after the eclipse, the pendulum experienced normal rotation, the Foucault effect, of $0.19^{\circ} /$ minute. $M$. Allais was not looking for any effect here [6].

He got similar results when he later repeated the experiment to a much lesser degree during a solar eclipse on October 2, 1959 (the amount of the solar surface eclipsed in Paris was only $36.8 \%$ of the surface eclipsed in 1954). Like in the first case a well-defined anomaly was detected in the motion of the paraconical pendulum: its plane of oscillation shifted abruptly. Both were partial eclipses in Paris, the point of observations. Currently accepted physical theory offers no explanation whatsoever for this phenomenon. His finding raises new questions about the nature of such phenomena.

Attempts to confirm Allais's observations upon the behavior of a pendulum during a solar eclipse have met with varied results: some experiments have confirmed the presence of anomalies, while some yielded ambiguous results, and others detected nothing unusual. However none of these trials used a paraconical pendulum according to Allais's design (hangs from a special joint that permits free rotation around the vertical and it can track the Earth's rotation); nor did the experimenters follow Allais's operational procedures or ask his advice on design of the experiments.

Most of this kind of experiments had been performed using other sorts of pendulums or with Foucault's pendulum which gives spectacular effects. Even if they are close, there are essential differences between the Foucault pendulum and the paraconical pendulum with anisotropic support. The paraconical pendulum is short, can turn on itself (capable of rolling in all directions upon a plane horizontal surface), was observed without discontinuity while Foucault pendulum is long, connected to the thread which supports it and has never been observed without discontinuity for the previous experiments. Although difficulties are inherent to a short Allais pendulum, and its movement is a complex phenomenon, difficult to analyze, as long as the pendulum oscillation remains flat, the movement in azimuth of the pendulum oscillation plane is reduced to 
the Foucault effect. The experiments of the paraconical pendulum with anisotropic support include totally the Foucault effect.

The deviation of the plane with regard to the plane corresponding to the Foucault effect, at most twenty minutes before the maximum of the eclipse, entailed increases of angular speeds corresponding to the fast variations of azimuth observed. In his book Anisotropie de PEspace [7], who is dedicated to the analysis of the eclipse effect in a more general context, M Allais quotes: "we can finally notice that the fast variations of azimuth observed from 11:20 am to 12 am and from 12:20 am to 1 pm correspond to angular speeds of the order of $6.2 \times 10^{-5}$ and $7.9 \times 10^{-5}$ radian per second which are respectively 1.13 and 1.43 times the Foucault effect (that is $5.5 \times 10^{-5}$ radian per second in the latitude of Saint-Germain's laboratory). The strengths involved in the noticed disturbances are thus of the order of magnitude of those who intervene in the Foucault effect".

This deviation due to the eclipse is interpreted as a kind of antigravitation. While for other pendulums (without free support) the antigravity will manifest itself by a change in the direction of rotation of the plane of oscillation and a lower angular speed of rotation (such as the torsion pendulum of Saxl and Allen in 1970 [8] which leads to the same conclusions as M. Allais), it will manifest itself in the case of the paraconical pendulum by a greater angular speed within the framework of a deviated plane.

The eclipse effect was again observed during the eclipse of the Sun on October 2, 1959. Later, at the University of Jassy (Romania), during the eclipse of the Sun on February 15, 1961, a sudden deviation from the oscillation plane of a Foucault pendulum was observed. The pendulum had oscillated in the same plane until the moment of the deflection. An experiment carried out in China during the 1973 eclipse seemed to confirm an Allais effect, but the experiment was not very conclusive because the protocol did not exclude many biases. Another experiment concerning the eclipse effect was carried out in Mexico City during the eclipse of July 11, 1991. After the maximum of the eclipse, a decrease in the rotational speed of the plane of oscillation was observed. During the total solar eclipse of August 11, 1999, the Allais effect was studied in Bucharest, using two Foucault pendulums. On the occasion of the eclipse, NASA (National Aeronautics and Space Administration) proposed a program to observe the effect. Several universities and laboratories around the world have participated in this program, coordinated by Dr. David Noever, but no in-depth analysis of the results was published. At the same time the phenomenon received the denomination of "Allais effect". Observations in Zambia and Australia between 2001 and 2002 also show anomalies. Eight gravimeters and two pendulums were deployed across six monitoring sites in China for the solar eclipse of July 22, 2009. Although one of the scientists involved described in an interview having observed the Allais effect, no result has been published in any academic journal [5] [6].

Observations of the Allais effect are rare and rather contradictory, not only because of the rarity of such eclipses, but also because no rigorous experimental protocol has been followed. The sometimes ambiguous experimental results 
mean that the veracity of the Allais effect remains controversial within the scientific community.

Regarding the experimental results for the eclipse experiment, let's say that since the total solar eclipse of May 29, 1919, proclaimed as the triumph of Einstein's theory, measurements of the deviation of light by the Sun were performed at a number of total eclipses. Although the measurements were difficult, the effect was very close to the predicted magnitude which is double the value obtained from Newtonian mechanics. But exact agreement between theory and measurement has never been achieved. The average of the observations made during eight eclipses between 1919 and 1960 gives a figure of 1.97 for the deviation. This figure, although considered to be one of the proofs of relativity, is higher than the number predicted by the calculation and in a proportion greater than the experimental errors. Several physicists seem to estimate that a deflection $10 \%$ greater than the theoretical value is due to observation errors. Rather, we think that this is a real insufficiency of Einstein's theory, and the connection we have made between the Allais effect and the arc residue only strengthens our understanding.

To establish a link between the Allais effect and the anomaly of the arc residues of General Relativity during total solar eclipses, we will not take into account the unexplained regularities of lunisolar periodicity discovered by M. Allais to examine only the sudden and unpredictable disturbance that occurred during the solar eclipse of June 30, 1954, when the Moon interposing itself between the Earth and the Sun acted as a screen against gravity. We will perform the unorthodox calculation which follows by treating the paraconical pendulum as if it were a simple pendulum.

\section{Mathematical Treatment}

\subsection{Gravitational Acceleration during the Disturbance Due to the Eclipse}

Let us take a simple pendulum in Paris $\left(49^{\circ} \mathrm{N}\right)$, which can be considered to be a point mass suspended from a string or rod of negligible mass, and suppose this resonant system with a single resonant frequency is with a free anisotropic support. For small amplitudes, the period of such a pendulum can be approximated by

$$
T=2 \pi(l / g)^{1 / 2}
$$

( 1 : length; gravitational acceleration $g$ in Paris is $9.8094 \mathrm{~m} / \mathrm{s}^{2}$ ) [9]. The time of a complete revolution of the oscillation plane around the vertical is

$$
T=2 \pi /(w \sin \theta)=24 h /\left(w \sin 49^{\circ}\right)=31.8 h .
$$

As the oscillation plane got up of $13.5^{\circ}\left(360^{\circ}+13.5^{\circ}=373.5^{\circ}\right)$ with the eclipse, we apply the rule of three to find the time which would take the pendulum in Paris to make the complete rotation of the oscillation plane around the vertical line. If $360^{\circ}=31.8$ hours, $373.5^{\circ}=32.9925 \mathrm{~h}$. The time would be prolonged of 
$32.9925-31.8=1.1925 h$, or $71.55 \mathrm{mn}$. It would mean that with the anomaly due to the eclipse (increase of $13.5^{\circ}$ ), the time in Paris would be 1.0375 times longer (32.9935/31.8).

Considering that the length 1 of the formula (1) remains the same, the gravitational acceleration due to the eclipse will be

$$
\frac{4 \pi^{2} l}{T_{\text {Paris with anomaly }}^{2}}=\frac{4 \pi^{2} T_{\text {Paris }}^{2} g_{\text {Paris }}}{4 \pi^{2} 1.0375 T_{\text {Paris }}^{2}}=\frac{g_{\text {Paris }}}{1.0375}=\frac{9.8094}{1.0375}=9.4548 \mathrm{~m} / \mathrm{s}^{2} .
$$

We notice a decline of the gravitational acceleration: $9.4548 \mathrm{~m} / \mathrm{s}^{2}$ instead of $9.8094 \mathrm{~m} / \mathrm{s}^{2}[9]$.

A decrease of the gravitational acceleration is equivalent to a longer range of the radius. We know that $9.8094 \mathrm{~m} / \mathrm{s}^{2}$ apply to the Earth's radius $6.3776 \times 10^{6} \mathrm{~m}$

$$
R_{E}=\left(G M_{E} / g\right)^{1 / 2}=\left(G 5.98 \times 10^{24} \mathrm{~kg} / 9.8094 \mathrm{~m} / \mathrm{s}^{2}\right)^{1 / 2} .
$$

Let us find the terrestrial radius equivalent to a gravitational acceleration of $9.4548 \mathrm{~m} / \mathrm{s}^{2}$ :

$$
R_{E}=\left(G M_{E} / g\right)^{1 / 2}=\left(G 5.98 \times 10^{24} \mathrm{~kg} / 9.4548 \mathrm{~m} / \mathrm{s}^{2}\right)^{1 / 2}=6.49608 \times 10^{6} \mathrm{~m} .
$$

The radius is $118,481 \mathrm{~m}\left(6.49608 \times 10^{6} \mathrm{~m}-6.3776 \times 10^{6} \mathrm{~m}\right)$. This implies, during the disturbance due to the eclipse, an addition of $118.481 \mathrm{~km}$ to the Earth's radius, which gives a gravitational acceleration of $9.4548 \mathrm{~m} / \mathrm{s}^{2}$ in Paris.

\subsection{Parallax}

The solar parallax in general is the difference in the apparent position of the Sun as seen from the Earth's centre and a point one Earth radius away, i.e., the angle subtended at the Sun by the Earth's mean radius. If the Sun is at the zenith (directly overhead) its parallax is 0 . The parallax is at maximum when the Sun is seen on the horizon and it is called the horizontal parallax. Solar parallax is very important since it indicates the Sun's distance from Earth. Foucault, after making a more accurate measurement of the velocity of light, determined from the aberration of star light that the solar parallax must be about 8.80". Michelson and Newcomb, using Foucault's method, found a more accurate velocity of light, which when combined with a better aberration value, gave a solar parallax of $8.80 " \pm 0.01 "$. The value of $8.80 "$ for the average equatorial horizontal parallax was adopted in Paris in 1896 by the "Conférence internationale des étoiles fondamentales". Simply put, the parallax of Sun is the angle ASE under which an observer at the centre of Sun would see the terrestrial radius (Figure 1) [10].

We will suppose that during the eclipse the centre of the Sun is the theoretical apparent position of the star. We already know the distance Sun-Earth which is $1.495 \times 10^{11} \mathrm{~m} . \mathrm{S}$ is the centre of Sun and the angle ASE is the horizontal solar parallax. Because this angle is so short, we can confuse, without sensible error, the Earth radius $r$ with the arc AE of 8.80 arcseconds (or 8.80"), which is part of a circumference with centre the centre $S$ of Sun and for radius the length $d$, average distance between the two centres.

The length of arc AE, compared with the entire circumference, is given by the 
proportion:

$$
\frac{\operatorname{Arc~} \mathrm{AE}}{2 \pi d}=\frac{8.80^{\prime \prime}}{360^{\circ}}=\frac{r}{2 \pi R_{S-E}}=\frac{6.3776 \times 10^{6} \mathrm{~m}}{2 \pi 1.495 \times 10^{11} \mathrm{~m}}=6.789 \times 10^{-6}
$$

The arc AE, or the parallax angle 8.80", is equivalent to the Earth radius $6.3376 \times 10^{6} \mathrm{~m}$, and to a gravitational acceleration of $9.8094 \mathrm{~m} / \mathrm{s}^{2}\left[\mathrm{~g}=G M / R_{T}^{2}\right]$. For the gravitational acceleration of $9.4548 \mathrm{~m} / \mathrm{s}^{2}$ at most of the disturbance of the eclipse, corresponding to a radius being $6.49608 \times 10^{6} \mathrm{~m}$, we shall obtain the parallax angle 8.9634" (if $6.3776 \times 10^{6} \mathrm{~m}=8.80^{\prime \prime}$ and $6.49608 \times 10^{6} \mathrm{~m}=x ; x=$ $8.9634 ")$. The angle would vary of $0.1634 "(8.9634 "-8.80 ")$.

If we reverse the parallax as if the Earth was the centre, and as if the radius of the Sun formed an arc length on the circumference having the distance Earth-Sun for radius, we find the same circumference with a tiny increase of the arc length (Figure 2). If we place 0.1634 arcsecond at the point $p$ of minimum approach of the Sun, the minimal distance of approach $d$, slightly superior to the radius of the Sun, would be exceeded. The point $p$ would pass at $p$, pushing away the straight line constituting the deviated trajectory of photons, so widening the angle which it makes with the not deviated trajectory of photons emitted by the distant star.

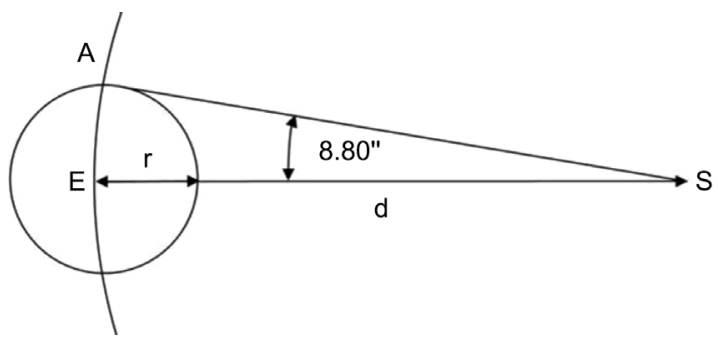

Figure 1. Parallax of the Sun. (E: Earth; S: Sun; r: radius of the Earth; d: the distance of the centers of the two stars; angle ESA: parallax, i.e., the angle under which an observer, placed in the center of the Sun, would see the terrestrial radius. According to the value adopted by the International Conference of Fundamental Stars held in Paris in 1896, the average equatorial horizontal parallax of the Sun is $8 " .80$ ).

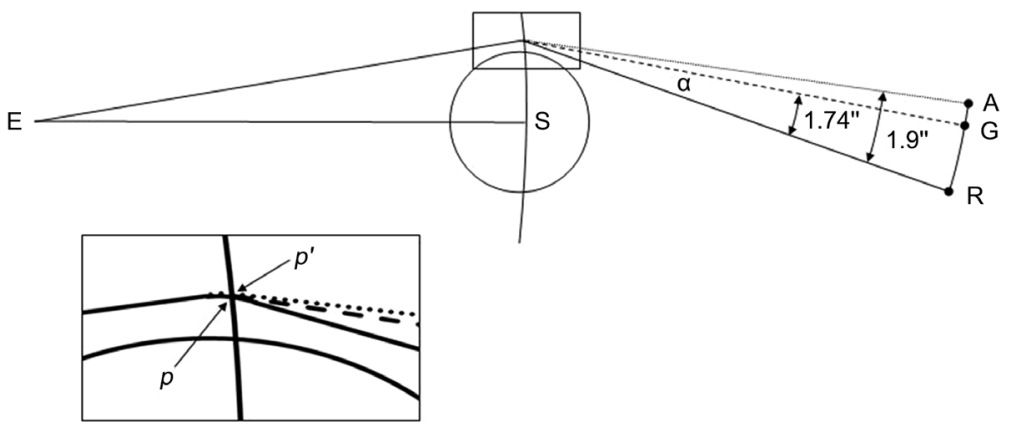

Figure 2. Parallax of the Earth during the solar eclipse where the Allais effect corresponds to the arc residue of General relativity. (E: Earth; S: Sun; R: real star with ray light; G: apparent star in accordance with GR with 1.74 arcseconds for the calculated angle RpG of deviation of rays of light; A: real observations of apparent star during total solar eclipses with $\sim 1.9 "$ for the observed angle Rp'A. The angular difference Gp'A coincides strangely with the brutal disturbance on the Allais pendulum during the total solar eclipse). 
This tiny increase of the arc length from $p$ to $p^{\prime}$, i.e. the angle $G p^{\prime} A$, constitutes a deviation about $10 \%$ of the theoretical value of the Relativity (if $1.7424 "=$ $100 \% ; 0.1634 "=9.37 \%$ ). We regard this as in satisfactory correspondence with the so-called residual arc, which have been tested by observations made at time of total eclipse on the apparent positions of stars whose light has passed close to the limb of the Sun [11].

\subsection{Value Calculated by General Relativity}

The value calculated by the theory of relativity of the angle of deviation of light by the Sun is

$$
\Delta=2 \alpha=4 G M_{S} / c^{2} R_{S}
$$

( $G$ is the universal constant of gravitation; $c$ is the speed of light, $M_{S}$ is the solar mass and $R_{S}$ is the Sun's radius [12]).

$$
\begin{aligned}
4 G M_{S} / c^{2} R_{S} & =4 G 1.98 \times 10^{30} \mathrm{~kg} /\left(c^{2} \sim 6.9535 \times 10^{8} \mathrm{~m}\right) \\
& =\left(8.4475 \times 10^{-6} \mathrm{rad}\right) \times 57^{\circ} 295 \times 3600^{\prime \prime}=\sim 1.7424^{\prime \prime}
\end{aligned}
$$

$2 \alpha$ indicates a deflection of light in the field of an attracting mass which is twice as great as would be calculated from the Newtonian theory for a particle travelling with the velocity of light, which is a huge progress. The factor 2 finds its origin in the existence of a temporal and "spatial" curvature in the metrics of Schwarzschild which represents the spacetime around the Sun. It allows verifying the existence of a deflection of light in passing through the gravitational field in the neighbourhood of the Sun, and to decide between Newtonian or Einsteinian theory [11].

\subsection{Value Calculated by the Theory of Relativity by Considering the Allais Effect}

The value calculated by the theory of relativity does not foresee nor explain the supplementary residual hundredth of arcsecond which were part of several measurements during eclipse of the bending of starlight by the Sun. No more than it foresees and explains the Allais effect during the total solar eclipse of 1954 when the Moon between Earth and Sun decreased the solar attraction.

The reckoning (6) giving 0.1634 arcsecond starts from a sudden disturbance on the Allais pendulum due to the total eclipse which reveals a decline of gravitational acceleration and consequently a length more remote from the terrestrial attractive centre. This added length, projected by means of the calculation of parallaxes on the circumference having for diameter the centres of the Sun and the Earth, is equivalent to the residual arc observed during experiments on the bending of light by gravity. Both observed phenomena, which arrive simultaneously only in eclipse time and are of the same magnitude, would be owed to the same cause: the antigravity provoked by the eclipse.

If we take into account the Allais disturbance and the residual arc observed during a total eclipse of the Sun, it will be necessary from the theory of relativity 
to consider that the point $p$ of minimum approach of the Sun is widened towards the outside and to add $\Delta \alpha$, the residual arc ensuing from the antigravity, to the angle of deviation $2 \alpha$. From an ad hoc point of view, purely observational, and only during eclipses, the Einsteinian formula could be:

$$
2 \alpha+\Delta \alpha=\left[4 G M_{S} / c^{2} R_{S}\right]+\Delta \alpha=1.7424 "+0.1634 "=\sim 1.90588 " .
$$

The angle does not correspond any more to the value calculated by the General theory of Relativity but rather to the average of the observations which gives a $10 \%$ deviation wider than the theoretical value $\left(1.74 "+\left(1.74^{\prime \prime} \times 10 \%\right)=\sim 1.916^{\prime \prime}\right)$ [13].

Let us underline that most of the experiments of eclipse collected results falling between 1.6" and 2.2". Experimenters found results below 1.74" (between $1.74 "$ and 1.6"), as if there was a kind of overgravity similar to the Pioneer effect. In that case the equation would become

$$
2 \alpha-\Delta \alpha=\left[4 G M_{S} / c^{2} R_{S}\right]-\Delta \alpha=1.7424 "-\Delta \alpha
$$

$\Delta \alpha$ being a fragment of arc going from $p$ towards the centre of the Sun, and which expresses a supplementary gravity due to the eclipse.

The formula of the General Relativity during total eclipse could thus be:

$$
2 \alpha \pm \Delta \alpha=\left[4 G M_{S} / c^{2} R_{S}\right] \pm \Delta \alpha .
$$

The three ad hoc formulae (9) (10) (11), although in compliance with the observations, remain nevertheless profoundly deficient not to say erroneous.

\subsection{Discussion}

According to General Relativity, a light ray passing in the vicinity of a celestial body undergoes a deflection in the direction of the decreasing gravitational potential, that is turned towards the celestial body itself, deflection of size $2 \alpha=4 G M_{S} / c^{2} R_{S}$. We suppose that the speed of light, given by

$$
\mathrm{d} s^{2}=g_{u v} \mathrm{~d} x_{u} \mathrm{~d} x_{v}=0
$$

equals 0 , and that $g_{44} / 2$, who plays the gravitational potential role determining the movement of the material point in a gravitational field stipulated almost static, does not vary [14]. At ordinary times, without eclipse, the interferometric experiments practically validated this formula in which $\alpha$ and $d$ are inversely proportional inside the point $p$ (Figure $2 \&$ Figure 3 ). With a more stressed curvature of light, the deflection angle $\alpha$ becomes wider as much as $d$, the minimal distance of approach of the centre of the Sun, gets closer to the length of the solar radius. It is the inverse for a lesser curvature.

During a total eclipse, our reckoning from the gravitational disturbance pin down by the pendulum gives a distance $d$ stretched out as well as a widened angle $2 \alpha$. They are proportional, what seems incomprehensible. By locating 0.1634 arcsecond at $p$, the minimal distance of approach of the centre of the Sun $d$, slightly superior to the Sun radius $R_{\mathcal{S}}$ is surpassed. Point $p$ placed at $p$, pushes 


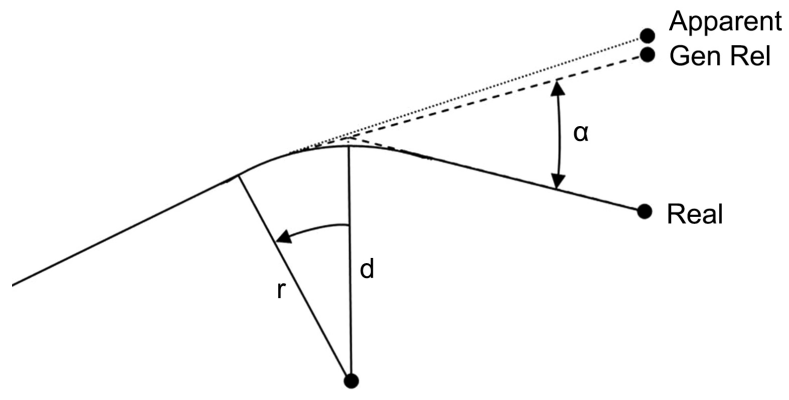

Figure 3. Deflection of a light ray in the vicinity of the Sun. ( $d$ is the minimum distance between the trajectory of the light ray and the centre of the Sun and is at a lightly upper distance than the Sun's radius $R_{S}$ )

away towards the outside the straight line forming the "deviated" trajectory of photons, so widening the angle which it makes with the "not deviated" trajectory of photons emitted by the distant star. This makes the formula inexplicable but the antigravity is implicit [13] [15].

Supposing that the pendulum got an excess of gravity, the calculation would have given a shorter distance $d$ corresponding to a smaller angle $2 \alpha$, that is going towards 1.6 arcseconds already observed during experiment of eclipse. Here also the distance $d$ would be proportional to the angle $2 \alpha$.

It is clear that the size $d$ observed during an eclipse is not the same as the theoretical value in normal time and is on average larger. We can suppose that the speed of light would not equal any more 0 (12) and that the gravitational potential can vary [15]. With the Allais effect of the 1954 eclipse, a ray of light passing along a celestial body would suffer a deflection on the side of the increasing gravitational potential, which is on the side opposed to the celestial body. The $g_{u v}$ would have varied in an unexpected way. There would have been, in addition to a concavity turned toward the Sun, which means that light rays curve with regard to the system of coordinates, a tiny concavity turned toward the outside, what indicates that starlight rays "straighten" [16]. It seems that from $p$ pointing toward $p$ 'the curvature of the space around a big mass such the Sun decreases and that a particle (planet or photon) is solicited according to the Newtonian laws. As if the gravitational potential $G M_{S} / R_{S}=c^{2}$ acquired higher potential during eclipse. As if $c^{2}$ becoming $v^{2}$ indicated an antigravity, meaning a lighter mass $M_{S}$ and an increasing "decurve" beyond the point $p$.

Certainly there is anomaly. The sudden proportionality between the angle $\alpha$ and the distance $d$ during the eclipse means a fundamental change in the interpretation of the expression $G M_{S} / c^{2}$. The factor $(2 \alpha+\Delta \alpha)$ plays a role as essential as $2 \alpha$ in the tests of the General Relativity and can only emerge on a new conception of the gravity.

\subsection{Formula of GR Altered during Solar Eclipses with Antigravitational Potential}

According to General Relativity, light emitted from a source far away from the 
Sun and passing near the Sun should be deflected by a theoretical $\sim 1.74$ arcseconds $(7,8)$, and the angle $\alpha$ is inversely proportional to $d$ the minimal distance of approach of the centre of the Sun.

Nevertheless, during total solar eclipse the observational angle is higher than the theoretical one and $d$ should be longer if there a real link between the eclipse and the lower gravity detected on Earth by the pendulum. So, if $\alpha$ and $d$ are higher, and the speed of lightstays the same, the solar mass should be higher. It seems impossible, unless there is an addition of a negative mass $\left(-\Delta M_{S}\right)$ which would act apparently as a positive mass. With $d$ higher, the trajectory of the ray of light is divergent outwards. Then the formula should be

$$
\begin{gathered}
2 \alpha_{o b s}=\left[4 G M_{S}\left(1+v^{2} / c^{2}\right)^{1 / 2}\right] /\left[c^{2} R_{S}\right] \\
2 \alpha_{o b s}=1.902^{\prime \prime}=\left[4 G 1.98 \times 10^{30} \mathrm{~kg}\left(1+v^{2} / c^{2}\right)^{1 / 2}\left(57^{\circ} .29578 \times 60^{\prime} \times 60 "\right)\right] \\
\div\left[c^{2}\left(6.9535 \times 10^{8} \mathrm{~m}\right)\right] \\
1.90588 " / 1.7424 "=1.09382461=\left(1+v^{2} / c^{2}\right)^{1 / 2} \\
v=\sim 1.33 \times 10^{8} \mathrm{~m} / \mathrm{s} .
\end{gathered}
$$

The Moon plays the role of a negative mass inducing an antigravity. And $v^{2}$ of $\left(1+v^{2} / c^{2}\right)^{1 / 2}$ could be considered as an antigravitational potential.

\section{Eclipse and Radio Interferometry Measurements}

We see that the numbers show that the Allais anomaly is in connection with the observed additional residual arcseconds and that both anomalies arise during a total solar eclipse.

Let us note on one hand that today the measure of these deflections is made by radio interferometry [1] [13] [16]. One of the advantages of this technique is that it can be made every year, by opposition to the measures of eclipse, which are sporadically taken and in inhospitable places.

The development of the interferometric methods in radio astronomy allowed verifying in a very precise way the predictions of Einstein and to impose strong limits on the possible anomalies of the General Relativity. On the other hand, it is strange that since 1919, while the best observations made by Eddington in favour of the value predicted by the General Relativity agreed in approximately $20 \%$ near, the measure of about four hundred stars during various eclipses did not allow to improve the precision of this method. The fact is that an exact agreement between theory and measurements has been obtained by radio interferometry but has never been obtained by eclipse technique [2]. The more effective measure with a precision of 0.05 of the bending of light made by radio interferometry inclines scientists to conclude that the General Relativity's value is confirmed, allows to release from eclipse experiences and end of story.

We conclude, on the contrary, that it rather confirms that there is an essential and intrinsic difference between the measures observed during eclipse and those 
without eclipse [13] [17] [18]. Our interpretation is that both anomalies result from the same phenomenon revealing an antigravity. The paraconical pendulum, of which the oscillation plane is free to turn all sides at the same time, seems to indicate that the more the anomaly increases the degree of oscillating plane (with regard to the plane corresponding to the Foucault effect), the more the deviated plane escapes the gravitation. The pendulum, and thus the Earth, is "lightened". Within the framework of the General Relativity, the excess of arcsecond would mean that there is "flatness", or a geodesic more remote from the Sun than the theoretical geodesic.

\section{Two Manners to Experiment}

Others could attribute it to an excess of gravity similar to the Pioneer effect [19]. Although the observational experiments are difficult it is important to compare experiments with total eclipse with other without eclipse for consistency. Fortunately, modern technology is available for such a comparison. The time delay can be measured as well as the arcseconds angles since the delay experienced by light passing a massive object is closely related to the deflection of starlight. Two manners thus offer to experiment to know if there is less or more gravity.

\subsection{Measure the Angle of Deflection of Light}

We could take the measure of light deflection near the Sun by using the radio interferometry on quasars. For some decades, the effect of deflection of the electromagnetic trajectories by a field of gravitation is determined by radio astronomers by using quasars 3C273 and 3C279. Quasars are, by definition, the best sources of radio energy. The positions of these two very close radio sources are exactly known and well placed to verify the deflection of radio energy by the Sun. On October 8th every year the movement of the Earth in orbit brings the Sun in the line with 3C279, darkening it. According to the prediction of Einstein, 3C279 disappears slightly later and reappears slightly earlier on the remote side from the Sun. The convenient position of $3 \mathrm{C} 373$ gives to radio astronomers a reference point to see how the apparent position of 3C279 is changed when it is on the edge of the Sun. Radio astronomers can see the separation angle with time, determine the deflection of light as a function of distance from the Sun and translate that into a deflection of a grazing ray. One can imagine that if ever such a heavenly coincidence could also coincide with a total solar eclipse, the photos taken by radio astronomers could be compared with those without eclipse. By careful analysis of the eclipses measurements they could see if there is in the sky an essential difference of the bending of light [1] [3] [17] [18].

\subsection{Measure Signal Delay}

Currently the deflection of "light" is best measured using radio astronomy, since radio waves can be measured during the day without waiting for an eclipse of the Sun. Einstein predicts that light will be delayed instead of accelerated when 
passing close to the Sun. By using the very precise radar techniques, we could measure the delay of the signals passing near the Sun during a total eclipse and to compare it with the delay obtained without eclipse. A radar impulse is sent from the Earth on a target of the solar system (a planet for example) which reflects this radio signal and sends it back on Earth. The measured round trip travel time of the radio wave has a slightly bigger value according to the General Relativity than according to Newton's theory. The difference is so much greater as the signal passes closer of the Sun. The first experiments used radar echo on planets Venus or Mercury in superior conjunction with the Sun: the wave radio cross, in that case, the field of solar gravitation on the way out and on the way back. It was matching the predicted amount of relativist time delay. The experiments have been repeated many times since, with increasing accuracy. This effect is now given by using a spacecraft behind the Sun instead of a star. Radar echo on space probes Mariner VI and VII who were placed on solar orbits after their observations of Mars, in 1969, gave delay observations which agree with Einstein within $\sim 0.9$ standard deviations. This was first done by Irwin Shapiro between 1966 and 1970 [19] [20] [21]. The duration of a radar signal during the eclipse should have shorter value than a delay observation without eclipse, and this shorter delay would go to the sense of an "antigravity". An additional delay during eclipse would mean "overgravity" similar to the Pioneer effect.

\section{Possible Cosmological Consequences}

We assume that there is a relation between the Allais effect and the not resolute anomaly of residual arc during total solar eclipse. It is evident at this stage that it needs further investigations in order to dissipate the confusion between significant gravitational anomaly and errors of observation, and determine if our calculation is indeed genuine or facility artefact. It must be taken into consideration that if the effects of these anomalies are real and in coincident evidence [22] [23], it gives a new aspect of the Allais effect which could lead at least to three possible cosmological consequences:

\subsection{If the Allais Effect Is Real, Gravity Would Be Attractive and Repulsive}

GR, based on the equivalence principle, is in an extension of special relativity and Newtonian gravity, but even if it is full of elegance and simplicity it doesn't mean that the theory is in agreement with recent observations (pioneer anomaly, anomaly of the astronomical unit, galaxy rotation curves, etc.) [24] [25]. If the Allais effect is real, gravitation could not be considered any more like a spacetime curvature or as only an attractive force. The element "antigravity" would put it to the rank of the other forces which have all an attractive and repulsive aspect. It would be then possible to describe gravity in the framework of quantum field theory like the other fundamental forces. 


\subsection{The Allais Effect on Cosmological Scales Would Apply to the Gravitational Lenses and to the Distribution of Matter}

It is easy to imagine that if the Allais effect is applied to the light-bending effect measured during a total eclipse of the Sun, then there is overwhelming evidence that this effect exists on various scales where eclipses are associated with light following curved paths through space distorted by the presence of matter.

According to GR, mass "warps" space-time to create gravitational fields and therefore bend light as a result. After this theory was confirmed in 1919 during a solar eclipse, Einstein realized that it was also possible for astronomical objects to bend light, and that under the correct conditions, one would observe multiple images of a single source, called a "gravitational lens" or sometimes a "gravitational mirage". It was not until 1979 that the first gravitational lens would be discovered. It became known as the "Twin Quasar" since it initially looked like two identical quasars. In the 1980s, astronomers realized that the combination of CCD imagers and computers would allow the brightness of millions of stars to be measured each night. Gravitational microlensing can provide information on comparatively small astronomical objects, such as Machos within our own galaxy, or extrasolar planets. Strong and weak gravitational lensing of distant galaxies by foreground clusters can probe the amount and distribution of mass, which is dominated by invisible dark matter. Aside from determining how much dark matter they contain, gravitational lensing can also be used to measure the expansion history of the Universe (its size as a function of time since the big bang), which is encoded in Hubble's law [1] [26] [27].

Einstein's GR demonstrates that a large mass can deform spacetime and bend the path of light. So, a very massive object, such as a cluster of galaxies can act as a gravitational lens (deflector). When light passes through the cluster from an object lying behind it, the light is bent and focused to produce an image or images of the source. Viewed from the observer (the Earth), the image may be magnified, distorted, or multiplied by the lens, depending upon the position of the source with respect to the lensing mass. So, let us suppose that a sufficiently visible or invisible massive object is moving between Earth and the deflector, or between the deflector and the source, would not there be an Allais effect?

If the data could be compared, before, during and after, that the massive object travels between us, the gravitational lens and the source, there could be an excessive residual arc of the optic angle due to the antigravity, a divergent angle showing itself by a little less brilliance than foreseen by the Relativity, different images. The Einstein effect, in times of eclipse, should have a slightly lower shift of the spectral lines towards the red (blueshift) than in times without eclipse. In case it would be an effect similar to the Pioneer effect (over gravity) we should have a slightly superior movement of the spectral lines towards the red than in a frame without eclipse.

Because on the surface of celestial bodies reigns a field of very intense gravitation and the movements of the lines are considerable, gravitational lensing for 
the generalized Relativity is a powerful tool to estimate mass distribution on cosmological scales. But if the additional Allais effect is true, the mass distribution and the interpretation of gravity could be different.

\subsection{Eclipse Perturbation Instead of Tidal Friction; Main Witness Becomes Main Culprit}

Universal time and the length of day would be subject to variations not only because of the tides, atmospheric circulation, internal effects, but also because of the Allais eclipse effect. Modern research shows that the Earth's rotation has slowed down by a fractional amount over the past 4000 years. The acceleration of the Moon's orbit would be associated with the deceleration of the Earth's rotation.

It is assumed that the Moon in its orbit experiences an outward accelerating effect caused by the friction of lunar and solar tides which slow the Earth's rotation. As the Earth rotates on its axis, it experiences the friction of the tides imposed by the gravitational pull of the Moon and, to a lesser extent, the Sun. This secular acceleration gradually transfers the angular momentum from the Earth to the Moon. As the Earth loses energy and slows down, the Moon gains this energy and its orbital period, as well as its distance from Earth, increases. Measurements over the past 40 years indicate that the Moon's orbit is receding from Earth at a rate of $4 \mathrm{~cm}$ per year.

It was on the basis of the Moon-Earth alignments at the time of the ancient eclipses that it was possible to conclude that-as a trend-the Earth's rotation is slowing down. Because a number of ancient eclipses have been collected, contemporary researchers have been able to determine that the daylength in the past was somewhat shorter than the current daylength $(86,400$ seconds). It seems that during the previous 4000 years the length of the day has gradually grown longer, and it is inferred that the ever increasing length of the day could be attributed to the decelerated rotation of the Earth. The observed lengthening of daylight, a change in the rate of rotation of -0.0018 seconds/century, across four millennia is found to have accumulated in the end a total increase of +0.07 seconds. This increase in the current day length of +0.07 seconds over the ancient days of 4000 years ago means that the rate of Earth's rotation has slowed down by -0.0018 seconds per century. While the indication that daylength has increased by a total of only 0.07 seconds over the past 4000 years may appear minimal, it is nonetheless significant; the accumulation of longer days, produced by a diminishing rotation, causes, after millennia, a noticeable change [28] [29].

It is thus assumed that the Moon in its orbit experiences an acceleration effect due to lunar and solar tides friction slowing down Earth's spin. The wave of tide lifting waters of oceans, moves the other way around of the rotation of the Earth; so it produces a friction which slows down the rotation. At the same time, the Moon undergoes a counteraction to that of the tide and goes away constantly from the Earth. However things seem more complicated than supposed. The 
vertical constituent of the force of Newton's static theory produces only an insignificant disturbance. The horizontal constituent of the force of the dynamic theory produces more impressive effects on the liquid masses but the influence of the oceanic friction can only be considered in the regions of weak depth and strong tides, what is the exception rather than the rule. Furthermore the atmospheric tides in resonance with the present period of rotation tend to accelerate the rotation and to compensate for the braking due to the oceanic tides. Thus no absolute conclusion can be formulated and it would be possible that this theory contributes only very partially to the slowing down of the Earth [14] [30] [31].

Our suggestion is that the slowing rotation of the Earth, the increasing length of the day associated to the wider lunar orbit, are above all caused by eclipses instead of tidal friction. In this respect we suggest to examine in depth the Allais eclipse effect [32]. It could reveal that mechanisms of "antigravity" could produce "cosmological leaps" and "cosmological leap seconds" (different from "leap seconds" filling the split between the Earth's rotation second and the atomic clock second) and be so the main cause of the deceleration in the Earth's spin rate and, simultaneously, of the receding of the Moon. The listed total solar eclipses were always the only historic witnesses of the non-uniform change of rate of the rotation of Earth and of the day length. More than witness of the changes in Earth's dynamical behaviour, we think that eclipse could be also considered as perpetrator if the eclipse records and the Allais effect were scientifically investigated. Eclipse would have acted like a pyromaniac who, having lit a long series of fires, hurries to indicate every fire to the authorities who, in turn, use him as unique witness for the prosecution against possible suspects.

\section{Conclusions}

Does the relationship between the Allais effect and the higher displacement than predicted by GR of the positions of star images during solar eclipse a simple hypothesis, or is it a consequence of the observation at the time of the total eclipse? We think that it is a consequence of the observational results at the time of the total eclipse and the measurements suggest that both anomalous phenomena have a common origin. Our approach to relating the two anomalies was first to calculate the gravitational acceleration in Paris during the disturbance caused by the total solar eclipse of June 30,1954, starting from the plane of oscillation of the paraconical pendulum of M. Allais which rose 13.5 degrees. Then find the Earth's radius equivalent to this abnormal gravitational acceleration. Using the average horizontal parallax of the Sun which is 8.80 " of arc, we find that the abnormal addition to the Earth's radius during the eclipse is equivalent to a parallax angle that would vary by $0.1634 "$. By reversing the parallax, we find the same circumference for the Sun with an excess of arc. It just so happens that 0.1634" corresponds to the deviation of about $10 \%$, that is to say to the arc residue verified during total eclipse experiments and which is beyond the experimental errors. 
Scientific history seems to want to repeat itself. When the GR predicted for the deflection of starlight just grazing the edge of the Sun an angular distance of 1.74 ", or two times the Newtonian prediction, the question of the precise value of the deviation became a matter of principle which had to allow choosing between both theories. The Relativity took it. Today, the Allais effect and the anomaly of residual arc during total solar eclipse persuade us that it is not the complete story. The observed 1.97" for the deviation is higher than the number predicted by the calculation and in a proportion superior to the experimental errors. And, as we tried to demonstrate, the unexplained arcseconds excess of these experiments would be in concomitance and accordance with the abrupt deviation of the plane of oscillation of the Allais pendulum with regard to the plane corresponding to the Foucault effect. GR does not explain the anomaly of the arc residue during total eclipses, which suggests that it is conceptually incomplete and that some substantive work needs to be done. We do not hesitate to assert that these confirmed experiences and these collected figures call into question the interpretation of GR and once again question our conception of the Universe.

\section{Conflicts of Interest}

The author declares no conflicts of interest regarding the publication of this paper.

\section{References}

[1] Bouquet, A. (1998) L’Univers de la Gravitation. Science \& Vie, No. 205, Hors-Série, 86-89.

[2] Born, M. (1962) Einstein's Theory of Relativity. Dover Publications Inc., Mineola, 358-359.

[3] Will, C.M. (1986) Was Einstein Right? Basic Books Inc., New York, 72-80, 82-86.

[4] Science@ NASA (1999) Decrypting the Eclipse. http://science.nasa.gov/newhome/headlines/ast06aug99_1

[5] Wikipedia, Allais Effect. http://en.wikipedia.org/wiki/Allais_effect

[6] Science du Monde (1969) La Gravité. Librairie Jules Tallandier et Nelson Doubleday Inc., 36-37.

[7] Allais, M. (1997) L’Anisotropie de l’Espace. Edition Clément Juglar, 91, 162-164, 171-175.

[8] Saxl, E.J. and Allen, M. (1971) Physical Review D, 3, 823. https://doi.org/10.1103/PhysRevD.3.823

[9] Resnick-Halliday (1979) Mécanique. Édition du renouveau pédagogique, 308, 309, 315, 362, 16-3 No. 6.

[10] Simard, H. (1916) Cours élémentaire de Cosmographie. Laflamme et Proulx, Quebec, 114-119.

[11] Tolman, R.C. (1987) Relativity Thermodynamics and Cosmology. Dover Publications, Inc., Mineola, 209-211.

[12] Rocard, J.-M. (1986) Newton et la Relativité, Que sais-je? Presse universitaire de 
France, Paris, 105-106.

[13] Pascoli, G. (1986) La Gravitation, Que sais-je? Presse universitaire de France, Paris, 43-45.

[14] Öpik, E.J. (1960) Initiation à l'astronomie, Petite Bibliothèque Payot. 66-67, 25-29, 39-40, 43-44.

[15] Balabar, F. (2002) Albert Einstein, Physique, Philosophie, Politique. Édition du Seuil, 326-327, 356-359, 363-365.

[16] Einstein, A. (1952) The Principle of Relativity. Dover Publications Inc., Mineola, 107-108.

[17] Mavridès, S. (1988) La Relativité, Que sais-je? Presse universitaire de France, Paris, 118-120.

[18] Nottale, L. (1994) L’Univers et la Lumière, Champs Flammarion. 188-190.

[19] Shapiro, I.I., et al. (1977) Journal of Geophysical Research, 82, 4329-4334. https://doi.org/10.1029/JS082i028p04329

[20] Calder, N. (1980) Einstein's Universe. Penguin Books, New York, 105-107.

[21] Wright, E.L. (2004) Deflection and Delay of Light. http://Astro.ucla.edu/ wright/deflection-delay.html

[22] Aujard, H. (2001) 21 st Century Science \& Technology, 14, 70. http://www.21stcenturysciencetech.com

[23] Duif, C.P. (2004) A Review of Conventional Explanations of Anomalous Observations during Solar Eclipses.

[24] Unzicker, A. (2008) Why Do We Still Believe in Newton's Law? Facts, Myths and Methods in Gravitational Physics.

[25] Amador, X.E. (2006) Review on Possible Gravitational Anomalies.

[26] Wikipedia, Gravitational Lenses. http://en.wikipedia.org/wiki/gravitational_lens

[27] Gribbin, J. and Rees, M. (1989) Cosmic Coincidences. Bantam Books, New York, 212-213.

[28] Steel, D. (1999) Eclipse. Headline Book Publishing, London, 141-152.

[29] Espenak, F. and Meeus, J. (2006) Five Millennium Canon of Solar Eclipses: -1999 to +3000. NASA Technical Publication TP-2006-214141. http://Eclipse.gsfc.NASA.gov/eclipse

[30] Bouteloup, J. (1968) Vagues, marées, courants marins, Que sais-je? Presses universitaires de France, Paris, 61-64.

[31] Rouch, J. (1961) Les Marées, Payot, Paris, Bibliothèque Scientifique, 70, 214-216.

[32] Oberg, E. (2007) Location of the Allais Effect on the Earth's Surface: A Hypothetical Field Model, Note 2. 